\title{
Radio observations of a sample of nearby BL Lacs
}

\author{
Gabriele Giovannini $^{1,2}$, Marcello Giroletti ${ }^{3}$ \\ and Greg B. Taylor ${ }^{4}$ \\ ${ }^{1}$ Dipartimento di Astronomia, via Ranzani 1, 40127 Bologna, Italy \\ email: ggiovannini@ira.cnr.it \\ ${ }^{2}$ Istituto di Radioastronomia, via Gobetti 101, 40129 Bologna, Italy \\ email: ggiovannini@ira.cnr.it \\ ${ }^{3}$ Dipartimento di Astronomia, via Ranzani 1, 40127 Bologna, Italy \\ email: mgirolet@ira.cnr.it \\ ${ }^{4}$ National Radio Astronomy Observatory, P.O. Box 0, Socorro, NM 87801, USA \\ email: gtaylor@nrao.edu
}

\begin{abstract}
We present a new sample of 30 nearby $(z<0.2)$ BL Lacs, selected to study the nuclear as well as the large scale properties of low power radio sources. New VLA and VLBA radio data have been obtained to discuss the morphology, physical properties, and overall properties of the sample. We show that the distributions of total radio power and intrinsic core radio power for BL Lacs are consistent with their being drawn from the same population as FR I radio galaxies.
\end{abstract}

\section{Introduction}

At present most systematic studies on the parsec (pc) and kpc scale of BL Lacs are based on the 1.Jy sample (Stickel et al. 1991). This sample consists mostly of powerful and distant BL Lac objects and could be not representative of general properties of BL Lacs. As for weaker objecs, similar studies are still missing or based on smaller numbers of objects observed only at arcsecond or milliarcsecond (mas) resolution. In order to investigate the general properties of BL Lacs with no selection effects on their intrinsic radio properties, we concentrated our attention to the sample discussed by Falomo et al. (2000) who studied the host galaxies of 30 nearby $(\mathrm{z}<0.2)$ BL Lacs with the Hubble Space Telescope (HST). The sample is not complete but no selection effect is present on the radio properties and it can be used to study the intrinsic radio properties and to discuss their parent population. Here we present preliminary results and refer to Giroletti (2004) and Giroletti et al. (2004) for a more detailed study. The availability of high quality data in the optical (HST) and in the radio band (VLA and VLBI) will allow a detailed comparison between nuclear and radio jets properties and the surrounding Interstellar Medium.

We assume $\mathrm{H}_{0}=70 \mathrm{~km} \mathrm{~s}^{-1} \mathrm{Mpc}^{-1}$

\section{Discussion}

From observational data we estimated the jet orientation and velocity and derived the Doppler factor for all sources in our sample. We used this result to compute the intrinsic core radio power. In Figure 1 we compare the intrinsic core radio power with the intrinsic core radio power estimated for a sample of FR I radio galaxies in the same redshift range by Giovannini et al. (2001). 

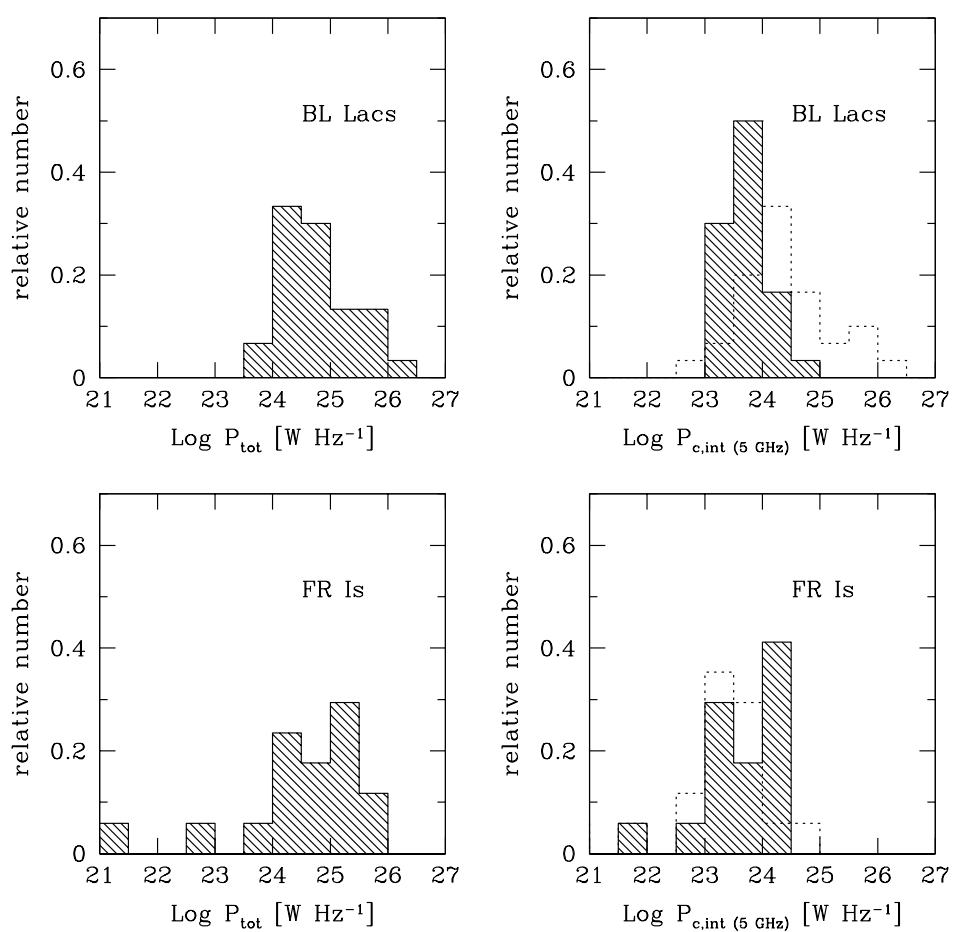

Figure 1. Distribution of total (left) and intrinsic core radio power (right) for objects in the present sample and FRI and low power radio galaxies studied by Giovannini et al. (2001). The dashed histograms overlayed to the intrinsic core radio power (right) show the distribution of the observed core radio power.

The two sample shows the same range in the total low frequency radio power (Fig. 1 left). We note that the low frequency total radio power is mainly related to the extended steep spectrum lobe radio emission expected to be not or marginally affected by Doppler boosting effects. In agreement with unified models where FR I radio galaxies are the parent population of BL Lacs, the intrinsic core radio power distribution in the two samples is also in agreement despite of the difference in the observed (dashed line) properties.

\section{Acknowledgements}

This work was partially support by the Italian Ministry for University and Research (MIUR) under grant COFIN 2002-028118 and 2003-027534. The National Radio Astronomy Observatory is operated by Associated Universities, Inc., under cooperative agreement with the National Science Foundation.

\section{References}

Falomo, R., Scarpa, R., Treves, A., \& Urry, C. M. 2000, ApJ, 542, 731

Giovannini, G., Cotton, W. D., Feretti, L., Lara, L., \& Venturi, T. 2001, ApJ, 552, 508

Giroletti, M. 2004, PhD Thesis, Astronomy Dept., Bologna University

Giroletti, M., Giovannini, G., Taylor, G. B., \& Falomo, R. 2004, ApJ, submitted

Stickel, M., Fried, J. W., Kuehr, H., Padovani, P., \& Urry, C. M. 1991, ApJ, 374, 431 